\title{
OBSERVABILITY AND CONTROLLABILITY OF THE 1-D WAVE EQUATION IN DOMAINS WITH MOVING BOUNDARY
}

\author{
ABDELMOUHCENE SENGOUGA
}

\begin{abstract}
By mean of generalized Fourier series and Parseval's equality in weighted $L^{2}$-spaces, we derive a sharp energy estimate for the wave equation in a bounded interval with a moving endpoint. Then, we show the observability, in a sharp time, at each of the endpoints of the interval. The observability constants are explicitly given. Using the Hilbert Uniqueness Method we deduce the exact boundary controllability of the wave equation.
\end{abstract}

\section{INTRODUCTION}

In this work, we will consider transverse oscillations of a uniform string whose length varies linearly with time. For $t \geq t_{0}>0$, we first denote

$$
\Omega_{t}:=(0, \ell t),
$$

which is an interval with the right endpoint depending on time. We assume that

$$
0<\ell<1,
$$

i.e. the length of $\Omega_{t}$ is increasing with a constant speed $\ell$ less then 1 . The case of a fixed interval, i.e. $\ell=0$, and intervals with a fast moving endpoint, $\ell \geq 1$, will not be considered here. We prefer to follow Balazs [2] and take the initial time $t_{0}>0$. We can take the initial time to be $t_{0}=0$ and an initial domain $\left(0, \ell_{0}\right)$, but this will complicate the mathematics and the Fourier formulas obtained below.

Let $T>t_{0}$ and consider the following non-cylindrical domain, and its lateral boundary,

$$
Q_{T}:=\bigcup_{t_{0}<s<T}\left\{\Omega_{s} \times\{s\}\right\}, \quad \Sigma_{T}:=\bigcup_{t_{0}<s<T}\left\{\partial \Omega_{s} \times\{s\}\right\} .
$$

Off course 1.1 ensure the so-called time-likeness condition $\left|\nu_{t}\right| \leq\left|\nu_{x}\right|$ on $\Sigma_{t}$, for $t>0$, where $\nu=\left(\nu_{t}, \nu_{x}\right)$ is the unit outward normal on the lateral boundary $\Sigma_{t}$. Let us now consider the following wave equation with homogeneous Dirichlet boundary conditions

$$
\begin{cases}\phi_{t t}-\phi_{x x}=0, & \text { in } Q_{T}, \\ \phi(0, t)=0, \quad \phi(\ell t, t)=0, & \text { for } t \in\left(t_{0}, T\right), \\ \phi\left(x, t_{0}\right)=\phi^{0}(x), \quad \phi_{t}\left(x, t_{0}\right)=\phi^{1}(x), & \text { for } x \in \Omega_{t_{0}}\end{cases}
$$

Date: April 19, 2018.

2010 Mathematics Subject Classification. 35L05, 93B05.

Key words and phrases. Wave equation, non-cylindrical domains, observability, controllability, Hilbert uniqueness method, generalized Fourier series. 
where $\phi(x, t)$ is the transverse displacement of the string and the subscripts $t$ and $x$ stand for the derivatives with respect to time and space, respectively. This is an example of evolution problems in non-cylindrical domains arising in many important applications (such as biology, engineering, quantum mechanics,...), see the survey paper by Knobloch and Krechetnikov [6].

Under the assumption (1.1), it is by now well known that for every initial data

$$
\phi^{0} \in H_{0}^{1}\left(\Omega_{t_{0}}\right), \phi^{1} \in L^{2}\left(\Omega_{t_{0}}\right)
$$

there exists a unique solution to Problem 1.2 such that

$$
\phi \in C\left(\left[t_{0}, T\right] ; H_{0}^{1}\left(\Omega_{t}\right)\right), \quad \phi_{t} \in C\left(\left[t_{0}, T\right] ; L^{2}\left(\Omega_{t}\right)\right),
$$

see [3, 9]. We define the "energy" of the above problem as

$$
E(t)=\frac{1}{2} \int_{0}^{\ell t} \phi_{x}^{2}+\phi_{t}^{2} d x \quad \text { for } t \geq t_{0} .
$$

which is not a conserved quantity in time, in contrast with the wave equation in cylindrical domains.

Let $\xi \in\{0, \ell t\}$ and denote $T_{0}:=T-t_{0}$. The problem of observability of (1.2) at the boundary $x=\xi$ can be formulated as follows: To give sufficient conditions on $T_{0}$ such that there exists $C\left(T_{0}\right)>0$ for which the following inequality holds for all solutions of 1.2 :

$$
E\left(t_{0}\right) \leq C\left(T_{0}\right) \int_{t_{0}}^{t_{0}+T_{0}} \phi_{x}^{2}(\xi, t)+\phi_{t}^{2}(\xi, t) d t .
$$

This is the so-called observability inequality, which allows estimating the energy of solutions in terms of the energy localized at the boundary $x=\xi$. The best value of $C\left(T_{0}\right)$ is the observability constant. Due to the finite speed of propagation (here equal to 1 ), the time $T_{0}$ should be sufficiently large and one expects that it depends on the initial length of $\Omega_{t_{0}}$ and also on the speed of expansion $\ell$.

On the other hand, we consider the following boundary controllability problem: Given

$$
\left(y^{0}, y^{1}\right) \in L^{2}\left(\Omega_{t_{0}}\right) \times H^{-1}\left(\Omega_{t_{0}}\right) \text { and }\left(y_{T}^{0}, y_{T}^{1}\right) \in L^{2}\left(\Omega_{t}\right) \times H^{-1}\left(\Omega_{t}\right),
$$

find a control function $v \in L^{2}\left(t_{0}, T\right)$, acting at the boundary $x=\xi$, such that the solution of

$$
\begin{cases}y_{t t}-y_{x x}=0, & \text { in } Q_{T}, \\ y(0, t)=\left(1-\frac{\xi}{\ell t}\right) v(t), \quad y(\ell t, t)=\frac{\xi}{\ell t} v(t), & \text { for } t \in\left(t_{0}, T\right), \\ y\left(x, t_{0}\right)=y^{0}(x), \quad y_{t}\left(x, t_{0}\right)=y^{1}(x), & \text { for } x \in \Omega_{t_{0}},\end{cases}
$$

satisfies

$$
y(T)=y_{T}^{0}, \quad y_{t}(T)=y_{T}^{1} .
$$

Problem (1.4) admits a unique solution in the sense of transposition, see [12, 11],

$$
y \in C\left(\left[t_{0}, T\right] ; L^{2}\left(\Omega_{t}\right)\right) \cap C^{1}\left(\left[t_{0}, T\right] ; H^{-1}\left(\Omega_{t}\right)\right) .
$$

To show the controllability, we use the Hilbert Uniqueness Method (HUM) introduced in the seminal work of Lions [10], see also Komornik [7]. The 
method reduces the controllability problem to the observability of the homogeneous problem (1.2).

The controllability of the wave equation in non-cylindrical domains was considered by several authors. Using the multiplier method, Bardos and Chen [3] derived some decay estimates for the wave equation in time-like domains, then show the exact internal controllability of the wave equation by a stabilization approach. Using a suitable change of variables, Miranda [12] transforms the non-cylindrical domain to a cylindrical one, obtaining a new operator with variable coefficients, then he shows the exact boundary controllability by HUM. Recently, there has been a renewed interest in such controllability problems. In particular, Problem (1.4) was considered, for instance, by [5, 4, 14] where the controllability is established by the multiplier method. Although this is a one-dimensional problem, no one so far, to my knowledge, gave the minimal time of controllability or observability and specified the constant of observability.

Going back to problems in cylindrical domains, the first results of observability and controllability of evolution problems was obtained by Fourier series, see Komornik and Loreti [8], Russell [13] and the references cited therein. This is not the case for non-cylindrical domains. To the author's knowledge, this paper is the first attempt to apply Fourier series techniques to establish observability and controllability results for the wave equation in non-cylindrical domains. Further results will be presented elsewhere.

In this work, we first express the solution of Problem 1.2 by a Fourier formula, then an estimate of the energy is derived by using Parseval's equality in a weighted $L^{2}$ space. In agreement with precedent works, for instance [14, the energy decays as $t^{-1}$. Next, we show that Problem 1.2 is observable at the fixed endpoint of the interval as well as at the moving one. The observability constants are explicitly given and obviously depend on $\ell$. The sharp time of observability, $T_{0}=2 \ell t_{0} /(1-\ell)$, turn out to be the same for both endpoints. Using HUM we obtain the exact boundary controllability, at one of the endpoints, of Problem (1.4) for $T_{0} \geq 2 \ell t_{0} /(1-\ell)$. This improves some recent results on the controllability of (1.4) obtained by the multiplier method. In particular, taking the initial length $\ell t_{0}=1$ and assuming that (1.1) holds,

- Cui et al. [5] showed the controllability of (1.4), at the fixed endpoint, for a larger time $T_{1}>\left(\exp \left(\frac{2 \ell(1+\ell)}{(1-\ell)^{3}}\right)-1\right) / \ell$.

- Sun et al. [14] showed the controllability of (1.4), at the moving endpoint, for $T_{2}>2 /(1-\ell)$. However, they did not address the limiting case $T_{2}=2 /(1-\ell)$ and its optimality. Cui et al. [4] obtained a larger time of controllability

$T_{3}>\left(\exp \left(\frac{2 \ell(1+\ell)}{(1-\ell)}\right)-1\right) / \ell$.

The remainder of this paper is organized as follows. In section 2 , we give the exact solution of Problem (1.2) then derive a sharp estimate for the energy. Next, the boundary observability and controllability at the fixed endpoint and at the moving endpoint are shown in the third and fourth section, respectively. 


\section{ENERgy estimates}

2.1. Exact solution. Let $a, b \in \mathbb{R}$, such that $a>b$ and consider a nonnegative $w:(a, b) \rightarrow \mathbb{R}$. In the sequel, we denote by $L^{2}(a, b, w d x)$ the weighted Hilbert space of measurable complex valued functions on $\mathbb{R}$, endowed by the scalar product

$$
\int_{a}^{b} f(x) \overline{g(x)} w(x) d x
$$

and its associated norm, see for instance Asmar [1. As usual, we drop $w d x$ in the space notation if $w \equiv 1$.

A well known result in analysis is that the set of functions $\left\{(1 / \sqrt{2}) e^{i n \pi z}\right\}_{n \in \mathbb{Z}}$ is a complete orthonormal set in the space $L^{2}(0,2)$. By making the change of variable

$$
z=\alpha_{\ell} \log \left(\frac{t+x}{t(1-\ell)}\right), \quad \text { for every } t \geq t_{0},
$$

where $\alpha_{\ell}=2 / \log \left(\frac{1+\ell}{1-\ell}\right)$, we obtain the set of function $\left\{\sqrt{\alpha_{\ell} / 2} e^{i n \pi \alpha_{\ell} \log (t+x)}\right\}_{n \in \mathbb{Z}}$ which is still a complete orthonormal set in the weighted Hilbert space $L^{2}\left(-\ell t, \ell t, \frac{d x}{(t+x)}\right)$. Note that 1.1 ensures that the weight function $1 /(t+x)$ is positive. By consequence, every $f(\cdot, t) \in L^{2}\left(-\ell t, \ell t, \frac{d x}{(t+x)}\right)$ can be written as

$$
f(x, t)=\sum_{-\infty}^{+\infty} c_{n} e^{i n \pi \alpha_{\ell} \log (t+x)}, \quad \forall t \geq t_{0}
$$

where the coefficients $c_{n}$ are given by

$$
c_{n}=\frac{\alpha_{\ell}}{2} \int_{-\ell t}^{\ell t} f(x, t) e^{-i n \pi \alpha_{\ell} \log (t+x)} \frac{d x}{(t+x)}, \quad \forall t \geq t_{0} .
$$

Moreover, the following Parseval's equality holds

$$
\int_{-\ell t}^{\ell t}|f(x, t)|^{2} \frac{d x}{(t+x)}=\left(\frac{2}{\alpha_{\ell}}\right) \sum_{-\infty}^{+\infty}\left|c_{n}\right|^{2}, \quad \forall t \geq t_{0} .
$$

Arguing as in [2], we can show that the exact solution of Problem (1.2), is the restriction to the interval $(0, \ell t)$ of the following function given by the generalized Fourier formulas

$$
\phi(x, t)=\sum_{-\infty}^{+\infty} C_{n}\left(e^{i n \pi \alpha_{\ell} \log (t+x)}-e^{i n \pi \alpha_{\ell} \log (t-x)}\right), \quad x \in(-\ell t, \ell t), t \geq t_{0} .
$$

Note that $\phi$ is an odd function of $x$ which ensure that the condition $\phi(0, t)=$ 0 is satisfied for every $t \geq t_{0}$. The coefficients $C_{n}$ are complex numbers, independent of $t$, given by

$$
C_{0}=0 \quad \text { and } \quad C_{n}=\frac{1}{4 n \pi i} \int_{-\ell t_{0}}^{\ell t_{0}}\left(\phi_{x}^{0}+\phi^{1}\right) e^{-i n \pi \alpha_{\ell} \log \left(t_{0}+x\right)} d x, \quad \text { if } n \neq 0
$$


where the initial conditions $\phi^{0}$ and $\phi^{1}$ are also considered as odd functions on $x$ defined on the interval $\left(-\ell t_{0}, \ell t_{0}\right)$.

2.2. Energy estimates. The following lemma gives the decay rate of the energy.

Lemma 1. Under the assumption (1.1), the solution of Problem (1.2) satisfies

$$
t E(t)+\int_{0}^{\ell t} x \phi_{x} \phi_{t} d x=S_{\ell}, \quad \text { for } t \geq t_{0}
$$

where $S_{\ell}:=2 \pi^{2} \alpha_{\ell} \sum_{-\infty}^{+\infty}\left|n C_{n}\right|^{2}$ is independent of $t$, and it holds that

$$
\frac{S_{\ell}}{(1+\ell) t} \leq E(t) \leq \frac{S_{\ell}}{(1-\ell) t}, \quad \text { for } t \geq t_{0} .
$$

Proof. First, we deduce from the exact formula of the solution that

$$
\begin{aligned}
& \phi_{t}(x, t)=\sum_{-\infty}^{+\infty} i n \pi \alpha_{\ell} C_{n}\left(\frac{e^{i n \pi \alpha_{\ell} \log (t+x)}}{(t+x)}-\frac{e^{i n \pi \alpha_{\ell} \log (t-x)}}{(t-x)}\right), \\
& \phi_{x}(x, t)=\sum_{-\infty}^{+\infty} i n \pi \alpha_{\ell} C_{n}\left(\frac{e^{i n \pi \alpha_{\ell} \log (t+x)}}{(t+x)}+\frac{e^{i n \pi \alpha_{\ell} \log (t-x)}}{(t-x)}\right),
\end{aligned}
$$

which are respectively an odd and an even functions of $x$, for every $t \geq t_{0}$. In particular we deduce that

$$
(t+x)\left(\phi_{x}+\phi_{t}\right)=2 \pi \alpha_{\ell} \sum_{-\infty}^{+\infty} i n C_{n} e^{i n \pi \alpha_{\ell} \log (t+x)} .
$$

Thanks to the Parseval's equality, applied to $(t+x)\left(\phi_{x}+\phi_{t}\right)$ as a function in the Hilbert space $L^{2}\left(-\ell t, \ell t, \frac{d x}{(t+x)}\right)$, we have

$$
\int_{-\ell t}^{\ell t}(t+x)\left(\phi_{x}+\phi_{t}\right)^{2} d x=\int_{-\ell t}^{\ell t}\left|(t+x)\left(\phi_{x}+\phi_{t}\right)\right|^{2} \frac{d x}{(t+x)}=\left(\frac{2}{\alpha_{\ell}}\right) 4 \pi^{2} \alpha_{\ell}^{2} \sum_{-\infty}^{+\infty}\left|n C_{n}\right|^{2}
$$

i.e.

$$
\int_{-\ell t}^{\ell t}(t+x)\left(\phi_{x}+\phi_{t}\right)^{2} d x=4 S_{\ell} \quad \text { for } t \geq t_{0}
$$

Both sides are finite since $\phi_{x}, \phi_{t} \in L^{2}(-\ell t, \ell t)$. Changing $x$ by $-x$ in the last formula, we also obtain

$$
\int_{-\ell t}^{\ell t}(t-x)\left(\phi_{x}-\phi_{t}\right)^{2} d x=4 S_{\ell}, \quad \text { for } t \geq t_{0} .
$$

Summing up 2.5 and (2.6), we infer that

$$
2 t \int_{-\ell t}^{\ell t} \phi_{x}^{2}+\phi_{t}^{2} d x+4 \int_{-\ell t}^{\ell t} x \phi_{x} \phi_{t} d x=8 S_{\ell}, \quad \text { for } t \geq t_{0}
$$


Since all the functions under the integral signs are even of $x$, then

$$
\frac{t}{2} \int_{0}^{\ell t} \phi_{x}^{2}+\phi_{t}^{2} d x+\int_{0}^{\ell t} x \phi_{x} \phi_{t} d x=S_{\ell}, \quad \text { for } t \geq t_{0},
$$

and 2.1 follows. To show 2.2, we use the inequality $\pm a b \leq a^{2}+b^{2}$ to obtain

$$
\pm \int_{0}^{\ell t} x \phi_{x} \phi_{t} d x \leq \ell t E(t), \quad \text { for } t \geq t_{0} .
$$

Taking into account (2.1), it comes that

$$
t(1-\ell) E(t) \leq S_{\ell} \quad \text { and } \quad t(1+\ell) E(t) \geq S_{\ell} .
$$

This implies (2.2) and the lemma is proved.

Remark 1. Since (2.7) holds also for $t=t_{0}$, then we get

$$
t(1-\ell) E(t) \leq t_{0}(1+\ell) E\left(t_{0}\right) \quad \text { and } \quad t(1+\ell) E(t) \geq t_{0}(1-\ell) E\left(t_{0}\right),
$$

for $t \geq t_{0}$. Thus the energy $E(t)$ satisfies

$$
\frac{(1-\ell) t_{0} E\left(t_{0}\right)}{(1+\ell) t} \leq E(t) \leq \frac{(1+\ell) t_{0} E\left(t_{0}\right)}{(1-\ell) t}, \quad \text { for } t \geq t_{0} .
$$

An analog inequality was obtained in [14, by the multiplier method.

\section{Observability and CONTROllability at the FIXed EndPoint.}

In this section, we show the observability of 1.2 at $\xi=0$, then by applying HUM we deduce the exact controllability of (1.4). First, we can state the following Lemma.

Lemma 2. Under the assumption (1.1), the solution of (1.2) satisfies

$$
\int_{t_{0}}^{\left(\frac{1+\ell}{1-\ell}\right)^{M} t_{0}} t \phi_{x}^{2}(0, t) d t=4 M S_{\ell}
$$

for any integer $M \geq 1$, and it holds that

$$
4 M t_{0}(1-\ell) E\left(t_{0}\right) \leq \int_{t_{0}}^{\left(\frac{1+\ell}{1-\ell}\right)^{M} t_{0}} t \phi_{x}^{2}(0, t) d t \leq 4 M t_{0}(1+\ell) E\left(t_{0}\right) .
$$

Proof. Taking $x=0$ in (2.4), we obtain

$$
t \phi_{x}(0, t)=2 \pi \alpha_{\ell} \sum_{-\infty}^{+\infty} i n C_{n} e^{i n \pi \alpha_{\ell} \log t} .
$$

Noting that

$$
e^{i n \pi \alpha_{\ell} \log \left(\left(\frac{1+\ell}{1-\ell}\right)^{M} t_{0}\right)}=e^{i n \pi \alpha_{\ell} \log t_{0}} \times e^{i n M \pi \alpha_{\ell} \log \left(\frac{1+\ell}{1-\ell}\right)}=e^{i n \pi \alpha_{\ell} \log t_{0}},
$$

then, we can check that $\left\{\sqrt{\alpha_{\ell} / 2 M} e^{i n \pi \alpha_{\ell} \log t}\right\}_{n \in \mathbb{Z}}$ is a complete orthonormal set in the space $L^{2}\left(t_{0},\left(\frac{1+\ell}{1-\ell}\right)^{M} t_{0}, \frac{d t}{t}\right)$ and by Parseval's equality it 
follows that

$$
\int_{t_{0}}^{\left(\frac{1+\ell}{1-\ell}\right)^{M} t_{0}} t \phi_{x}^{2}(0, t) d t=\int_{t_{0}}^{\left(\frac{1+\ell}{1-\ell}\right)^{M} t_{0}} t^{2} \phi_{x}^{2}(0, t) \frac{d t}{t}=\left(\frac{2 M}{\alpha_{\ell}}\right) 4 \pi^{2} \alpha_{\ell}^{2} \sum_{-\infty}^{+\infty}\left|n C_{n}\right|^{2}
$$

which implies (3.1). The estimate (3.2) follows by using (2.7) for $t=t_{0}$.

Remark 2. From (3.2) we infer that

$$
\int_{t_{0}}^{\left(\frac{1+\ell}{1-\ell}\right)^{M} t_{0}} \phi_{x}^{2}(0, t) d t \leq 4 M(1-\ell) E\left(t_{0}\right) .
$$

Then, for any $T \geq t_{0}$, we can always choose an integer $M$ such that $T \leq$ $\left(\frac{1+\ell}{1-\ell}\right)^{M} t_{0}$ and since the integrated function is nonnegative, we deduce the following (so-called direct) inequality

$$
\int_{t_{0}}^{T} \phi_{x}^{2}(0, t) d t \leq 4 M(1-\ell) E\left(t_{0}\right) .
$$

Noting that $\phi_{t}(0, t)=0, \forall t \geq t_{0}$, inequality 1.3$)$ reads

$$
E\left(t_{0}\right) \leq C\left(T_{0}\right) \int_{t_{0}}^{t_{0}+T_{0}} \phi_{x}^{2}(0, t) d t
$$

hence Lemma 2 is very useful to establish the observability of $(1.2)$ at $\xi=0$.

Theorem 1. Under the assumption (1.1), if $T_{0} \geq 2 \ell t_{0} /(1-\ell)$, Problem (1.2) is observable at the fixed endpoint $\xi=0$ and it holds that

$$
E\left(t_{0}\right) \leq \frac{1+\ell}{4(1-\ell)^{2}} \int_{t_{0}}^{t_{0}+T_{0}} \phi_{x}^{2}(0, t) d t
$$

Conversely, if $T_{0}<2 \ell t_{0} /(1-\ell),(1.2)$ is not observable at $\xi=0$.

Proof. Noting that $\frac{(1+\ell) t_{0}}{(1-\ell)}=t_{0}+\frac{2 \ell t_{0}}{(1-\ell)}$ and taking $M=1$ in 3.2 , then we get

$$
4 t_{0}(1-\ell) E\left(t_{0}\right) \leq \frac{1+\ell}{1-\ell} t_{0} \int_{t_{0}}^{t_{0}+\frac{2}{1-\ell} \ell t_{0}} \phi_{x}^{2}(0, t) d t
$$

and thus inequality (3.5) holds for $T_{0}=2 \ell t_{0} /(1-\ell)$ and therefore for any $T_{0}>2 \ell t_{0} /(1-\ell)$ as well.

To show that the observability does not hold for $T_{0}<2 \ell t_{0} /(1-\ell)$, we adapt a proof found in [15] where the wave equation is considered in a fixed interval. Set $T_{\delta}=\frac{(1+\ell) t_{0}-2 \delta}{1-\ell}$ for some $\delta>0$ sufficiently small, then solve

$$
\begin{cases}u_{t t}-u_{x x}=0, & \text { in } Q_{T}, \\ u(0, t)=0, \quad u(\ell(t), t)=0, & \text { for } t \in\left(t_{0}, T_{\delta}\right), \\ u(x, s)=u^{0}(x), \quad u_{t}(x, s)=u^{1}(x), & \text { for } x \in \Omega_{s},\end{cases}
$$

with data at time $s=\left(t_{0}-\delta\right) /(1-\ell)$ with support in the subinterval $(\ell s-\delta, \ell s)$, (see Figure 1a). Let us check that the solution of $(3.6)$ is unique. In one hand, $u$ is unique for $t \geq s$ as it satisfy a wave equation, in an interval expanding at a speed $\ell<1$, with initial data at $t=s$. On the other hand, setting

$$
U(t)=u(s-t), \quad \text { for } t \in\left(t_{0}, s\right),
$$


then $U$ is unique as it satisfy a wave equation, in an interval contracting at a speed $\ell<1$, with initial data (see [3])

$$
U(x, 0)=u^{0}(x), \quad U_{t}(x, 0)=u^{1}(x) \quad \text { in }(0, \ell s) .
$$

Thus $u$ is uniquely determined for $t \geq t_{0}$. This solution is such that $u_{x}(0, t)=$ 0 for $t_{0}+\delta<t<T_{\delta}-\delta$ since the segment $x=0, t \in\left(t_{0}+\delta, T_{\delta}-\delta\right)$ remains outside the domain of influence of the segment $t=s, x \in(\ell s-\delta, \ell s)$. This means that

$$
\int_{t_{0}+\delta}^{T_{\delta}-\delta} \phi_{x}^{2}(0, t) d t=0, \quad \forall \delta>0,
$$

and the observability inequality (3.5) does not hold.



(a)



(b)

Figure 1. Propagation of a wave with a small support near an endpoint

Remark 3. An initial disturbance concentrated near $x=0$ may propagate to the right, as $t$ increases, and bounce back on the moving boundary, when $t$ is close to $\frac{t_{0}}{1-\ell}$, then travel to the left to reach the fixed boundary, when $t$ is close to $\frac{1+\ell}{1-\ell} t_{0}$, (see Figure 1a). Thus the needed time to complete this journey is close to $\frac{(1+\ell) t_{0}}{1-\ell}-t_{0}=2 \ell t_{0} /(1-\ell)$, which is the critical time of observability.

Remark 4. Let us fix the initial length $t_{0}=1$ (by choosing $t_{0}=1 / \ell$ ). Then, as $\ell \rightarrow 0$, we recover the critical time of observability $T_{0}=2$ of the wave equation in the fixed interval $(0,1)$.

The idea of HUM is based on the equivalence between the observability at $\xi=0$ of the homogeneous problem 1.2 and the exact controllability at $\xi=0$ of the non-homogeneous problem (1.4). The proof of this equivalence for the wave equation in an interval with fixed ends (see, for instance, pages 53-57 of [7]) can be carried out without much difficulty to yield the same result for an interval with moving ends. Whence we have the following controllability result. 
Corollary 1. Under the assumption (1.1), Problem (1.4) is exactly controllable at the fixed endpoint $\xi=0$ for $T_{0} \geq 2 \ell t_{0} /(1-\ell)$. Moreover, we can choose the control $v$ satisfying

$$
\int_{t_{0}}^{t_{0}+T_{0}} v^{2}(t) d t \leq K\left(T_{0}, \ell\right) E\left(t_{0}\right)
$$

where $K\left(T_{0}, \ell\right)$ is a constant depending on $T_{0}$ and $\ell$.

Conversely, if $T_{0}<2 \ell t_{0} /(1-\ell),(1.4)$ is not controllable at $\xi=0$.

Remark 5. The control obtained by HUM is $v=\phi_{x}(0, t)$, where $\phi$ is the solution of (1.2) with some suitable choice of the initial conditions, see also the proof of Theorem 2.1 in [5]. Inequality (3.7) is a consequence of (3.4).

\section{Observability and CONTROllability at the MOVing EndPoint.}

In this section, we show the observability and the controllability at $\xi=\ell t$. Let us start with the following lemma.

Lemma 3. Under the assumption (1.1), the solution of (1.2) satisfies

$$
\int_{t_{0}}^{\left(\frac{1+\ell}{1-\ell}\right)^{M} t_{0}} t \phi_{x}^{2}(\ell t, t) d t=\frac{4 M}{\left(1-\ell^{2}\right)^{2}} S_{\ell}
$$

for any integer $M \geq 1$, and it holds that

$$
\frac{4 M t_{0}}{(1+\ell)^{2}(1-\ell)} E\left(t_{0}\right) \leq \int_{t_{0}}^{\left(\frac{1+\ell}{1-\ell}\right)^{M} t_{0}} t \phi_{x}^{2}(\ell t, t) d t \leq \frac{4 M t_{0}}{(1-\ell)^{2}(1+\ell)} E\left(t_{0}\right) .
$$

Proof. Taking $x=\ell t$ in $(2.4)$, we get

$$
\phi_{x}(\ell t, t)=\pi \alpha_{\ell} \sum_{-\infty}^{+\infty} i n C_{n}\left(\frac{e^{i n \pi \alpha_{\ell} \log (1+\ell)}}{(1+\ell)}+\frac{e^{i n \pi \alpha_{\ell} \log (1-\ell)}}{(1-\ell)}\right) \frac{e^{i n \pi \alpha_{\ell} \log t}}{t},
$$

i.e.

$$
t \phi_{x}(\ell t, t)=\frac{2 \pi \alpha_{\ell}}{\left(1-\ell^{2}\right)} \sum_{-\infty}^{+\infty}\left(i n C_{n} e^{i n \pi \alpha_{\ell} \log (1+\ell)}\right) e^{i n \pi \alpha_{\ell} \log t}
$$

Then, by Parseval's equality in $L^{2}\left(t_{0},\left(\frac{1+\ell}{1-\ell}\right)^{M} t_{0}, \frac{d t}{t}\right)$ applied to the function $t \phi_{x}(\ell t, t)$, we infer that

$$
\int_{t_{0}}^{\left(\frac{1+\ell}{1-\ell}\right)^{M} t_{0}} t \phi_{x}^{2}(\ell t, t) d t=\left(\frac{2 M}{\alpha_{\ell}}\right) \frac{4 \pi^{2} \alpha_{\ell}^{2}}{\left(1-\ell^{2}\right)^{2}} \sum_{-\infty}^{+\infty}\left|n C_{n}\right|^{2}
$$

which implies (4.1). The estimate (4.2) follows by using (2.7) for $t=t_{0}$.

Remark 6. Inequality (4.2) also yields

$$
\int_{t_{0}}^{\left(\frac{1+\ell}{1-\ell}\right)^{M} t_{0}} \phi_{x}^{2}(\ell t, t) d t \leq \frac{4 M}{(1-\ell)^{2}(1+\ell)} E\left(t_{0}\right) .
$$


For any $T \geq t_{0}$, taking $M$ such that $T \leq\left(\frac{1+\ell}{1-\ell}\right)^{M} t_{0}$, we have the following direct inequality

$$
\int_{t_{0}}^{T} \phi_{x}^{2}(\ell t, t) d t \leq \frac{4 M}{(1-\ell)^{2}(1+\ell)} E\left(t_{0}\right) .
$$

Since $\phi(\ell t, t)=0, \forall t \geq t_{0}$, then $\phi_{t}(\ell t, t)+\ell \phi_{x}(\ell t, t)=0, \forall t \geq t_{0}$. In this case, inequality $(1.3)$ reads

$$
E\left(t_{0}\right) \leq C^{\prime}\left(T_{0}\right)\left(1+\ell^{2}\right) \int_{t_{0}}^{t_{0}+T_{0}} \phi_{x}^{2}(\ell t, t) d t .
$$

Thus Lemma 3 can be used to show the observability at $\xi=\ell t$.

Theorem 2. Under the assumption (1.1), if $T_{0} \geq 2 \ell t_{0} /(1-\ell)$, Problem (1.2) is observable at the moving endpoint $\xi=\ell t$ and it holds that

$$
E\left(t_{0}\right) \leq \frac{(1+\ell)^{3}}{4} \int_{t_{0}}^{t_{0}+T_{0}} \phi_{x}^{2}(\ell t, t) d t .
$$

Conversely, if $T_{0}<2 \ell t_{0} /(1-\ell),(1.2)$ is not observable at $\xi=\ell t$.

Proof. Setting $M=1$ in 4.2 , we infer

$$
\frac{4 t_{0}}{(1+\ell)^{2}(1-\ell)} E\left(t_{0}\right) \leq \frac{1+\ell}{1-\ell} t_{0} \int_{t_{0}}^{\frac{1+\ell}{1-\ell} t_{0}} \phi_{x}^{2}(\ell t, t) d t
$$

and (4.4) holds when $T_{0} \geq 2 \ell t_{0} /(1-\ell)$.

To show that (4.4) does not hold for $T_{0}<2 \ell t_{0} /(1-\ell)$, we argue as above. Consider again $T_{\delta}=\frac{(1+\ell) t_{0}-2 \delta}{1-\ell}$ for some $\delta>0$ sufficiently small. Solve Problem (3.6) with data at time $s=(1+\ell) t_{0}-\delta$ with support in the subinterval $(0, \delta)$, (see Figure 1b). This solution is such that $u_{x}(\ell t, t)=0$ for $t_{0}+\delta<t<T_{\delta}-\delta$ since the segment $x=\ell t, t \in\left(t_{0}+\delta, T_{\delta}-\delta\right)$ remains outside the domain of influence of the space segment $t=s, x \in(0, \delta)$, hence

$$
\int_{t_{0}+\delta}^{T_{\delta}-\delta} \phi_{x}^{2}(\ell t, t) d t=0, \quad \forall \delta>0 .
$$

This ends the proof.

Remark 7. Although the observability constant is different in (3.5) and (4.4), but the observability time is the same $T_{0}=2 \ell t_{0} /(1-\ell)$. As in Remark 3, this value can be justified by using characteristics, (see Figure 1b).

Arguing as in the precedent section, we have the following controllability result.

Corollary 2. Under the assumption (1.1), Problem (1.4) is exactly controllable at the moving endpoint $\xi=\ell$ for $T_{0} \geq 2 \ell t_{0} /(1-\ell)$. Moreover, we can choose the control $v$ satisfying

$$
\int_{t_{0}}^{t_{0}+T_{0}} v(t) d t \leq K^{\prime}\left(\ell, T_{0}\right) E\left(t_{0}\right)
$$

where $K^{\prime}\left(T_{0}, \ell\right)$ is a constant depending on $\ell$ and $T_{0}$.

Conversely, if $T<2 \ell t_{0} /(1-\ell),(1.4)$ is not controllable at $\xi=\ell t$. 
Remark 8. The control obtained by HUM is $v=\phi_{x}(\ell t, t)$, with some suitable choice of the initial conditions in (1.2), see also the proof of Theorem 1.2 in [14]. Inequality (4.5) is a consequence of (4.3).

\section{REFERENCES}

[1] N. Asmar. Partial differential equations with Fourier series and boundary value problems. Prentice Hall, 2005.

[2] N. Balazs. On the solution of the wave equation with moving boundaries. J. Math. Anal. Appl., 3:472-484, 1961.

[3] C. Bardos and G. Chen. Control and stabilization for the wave equation. III: Domain with moving boundary. SIAM J. Control Optim., 19:123-138, 1981.

[4] L. Cui, X. Liu, and H. Gao. Exact controllability for a one-dimensional wave equation in non-cylindrical domains. J. Math. Anal. Appl., 402:612-625, 2013.

[5] L. Cui, Y. Jiang, and Y. Wang. Exact controllability for a one-dimensional wave equation with the fixed endpoint control. Bound. Value Probl., 2015(1):1-10, 2015.

[6] E. Knobloch and R. Krechetnikov. Problems on time-varying domains: Formulation, dynamics, and challenges. Acta Applicandae Math., 137(1):123-157, dec 2014.

[7] V. Komornik. Exact Controllability and Stabilization. The multiplier method, volume 36 of RMA. Masson-John Wiley \& Sons, 1994.

[8] V. Komornik and P. Loreti. Fourier series in control theory. Springer, 2005.

[9] J. L. Lions. Quelques méthodes de résolution des problèmes aux limites non linéaires. Dunod-Gautier Villars, 1969.

[10] J.-L. Lions. Contrôlabilité exacte, stabilisation et perturbations de systemes distribués. Tome 1. Contrôlabilité exacte, volume 8 of RMA. Masson, 1988.

[11] L. A. Medeiros, M. M. Miranda, and A. T. Lourdo. Introduction to exact control theory: Method HUM. Editora da Univ. Estadual da Paraba, 2013.

[12] M. M. Miranda. Exact controllability for the wave equation in domains with variable boundary. Rev. Mat. Complut., 9(2), 1996.

[13] D. L. Russell. Controllability and stabilizability theory for linear partial differential equations: recent progress and open questions. SIAM Rev., 20(4):639-739, 1978.

[14] H. Sun, H. Li, and L. Lu. Exact controllability for a string equation in domains with moving boundary in one dimension. Electron. J. Diff. Equations, 98:1-7, 2015.

[15] E. Zuazua. Propagation, observation, and control of waves approximated by finite difference methods. SIAM Rev., 47(2):197-243, 2005.

(Abdelmouhcene Sengouga) Laboratory of Functional Analysis and Geometry of Spaces, University of M'sila, 28000 M'sila, Algeria.

E-mail address: amsengouga@gmail.com 